\title{
Book Review: Modern Poisons: A Brief Introduction to Contemporary Toxicology
}

\author{
Jeffrey M. Carlson * \\ School of Behavioral and Social Sciences, Arizona State University, Glendale, CA, USA
}

Keywords: toxins, dose-response, endocrine disruption, pesticides, chemical resistance, epigenetics

\author{
A book review on \\ Modern Poisons: A Brief Introduction to Contemporary Toxicology \\ Edited by Alan S. Kolok, Washington: DC: Island Press, 2016. pp. 1-208. \\ ISBN: 9781610913829
}

OPEN ACCESS

Edited by:

Abdel-Tawab H. Mossa,

National Research Centre, Egypt

Reviewed by:

Christian Sonne,

Aarhus University, Denmark

Eman Saed Swelam,

Cairo University, Egypt

${ }^{*}$ Correspondence:

Jeffrey M. Carlson jcarlso20@gmail.com

Specialty section:

This article was submitted to

Environmental Toxicology,

a section of the journal

Frontiers in Environmental Science

Received: 01 August 2016

Accepted: 15 August 2016

Published: 26 August 2016

Citation:

Carlson JM (2016) Book Review: Modern Poisons: A Brief Introduction

to Contemporary Toxicology.

Front. Environ. Sci. 4:55

doi: 10.3389/fenvs.2016.00055
The 1900s witnessed multitudinous advances in the fields of medicine and technology. Paradoxically, this progress resulted in both the prolongation of life expectancy and increased variability in factors contributing to morbidity and mortality. Currently, the sensationalized media coverage surrounding environmentally-driven health outcomes is disconnected from the underlying academic literature. In Modern Poisons, Alan S. Kolok, the Director of the Center for Environmental Health and Toxicology at the University of Nebraska, utilizes his 20-plus years in the field to bridge this divide. Kolok's book examines and historically situates toxicology as a discipline and provides a credible and accessible account of present day themes and approaches within the field.

Kolok begins by introducing and elaborating on concepts foundational to toxicology such as the dose-response curve and the relation between solubility to toxicity, a connection that admittedly took him some time to realize. He then summarizes the three main routes of contaminant absorption: skin, lungs, and digestive tract and lends insight into the body's physiological defense responses, sequestration and biotransformation. Importantly, Kolok presents an account of the mechanisms and cyclical nature of chemical and biological transport through the environment to elucidate and explain contaminant ubiquity and the subsequent relevance to individuals worldwide.

The book then journeys through various classes of chemicals, from naturally occurring poisons and venoms to anthropogenic pesticides and pharmaceuticals. Along the way, Kolok focuses on the conditions giving rise to their prevalence as well as the process by which they result in adverse health outcomes to an individual directly, and their offspring transgenerationally. Further, he shatters the misconception that low-dose exposures pose minimal health risk by describing the indiscriminate and transistor-like process of hormonal signaling as well as touching on the processes of bioconcentration and synergism. Throughout this process he carefully inserts information suggestive of dysfunctional regulatory oversight and a shifting of the burden proof to opponents of such contaminants.

While the author adeptly and passionately utilizes personally recognizable and relevant examples to facilitate a greater understanding of the concepts and methodological concerns of the field of toxicology, sections get bogged down in overly scientific technicality. Despite this, Kolok 
most often time treads the fine line between under explained and exceedingly scholarly wonderfully, filling a necessary void in environmental health and toxicology literature. The major success of this work lies in its ability to impart a fascination in the field while simultaneously situating itself as scientifically grounded.

Finally, the book provides a starting point for individuals interested in the field, enabling them to more easily traverse the toxicological landscape. Overall, the book should be incorporated into upper level undergraduate or graduate coursework as an introductory or exploratory piece that contributes a level of sophistication beyond surface-level, pop culture toxicology.

\section{AUTHOR CONTRIBUTIONS}

JC is the sole author and contributor to the book review.

Conflict of Interest Statement: The author declares that the research was conducted in the absence of any commercial or financial relationships that could be construed as a potential conflict of interest.

Copyright (๑) 2016 Carlson. This is an open-access article distributed under the terms of the Creative Commons Attribution License (CC BY). The use, distribution or reproduction in other forums is permitted, provided the original author(s) or licensor are credited and that the original publication in this journal is cited, in accordance with accepted academic practice. No use, distribution or reproduction is permitted which does not comply with these terms. 